\title{
Das Problem der Geschichtsphilosophie.
}

Eine Einführung in den systematischen Zusammenhang ihrer Probleme.

Von Fritz Münch-Jena. ${ }^{1}$ )

Die gesamte Philosophie der Gegenwart, soweit sie auf wissenschaftliches Gepräge Anspruch erheben kann, ist charakterisiert dadurch, dass jeder sachlichen Behandlung der Probleme irgend eines philosophischen Spezialgebietes eine erkenntnistheoretische Selbstbesinnung der auf dieses Gebiet gerichteten Untersuchungen vorauszugehen hat. Dieser Zug in dem philosophischen Bewusstsein unserer Zeit ist eine Konsequenz der von Kants methodus transzendentalis philosophandi vollzogenen kopernikanischen Wendung in der Stellung des Philosophen zurWirklichkeit überhaupt, und damit auch in der Stellung der philosophischen Probleme selbst. Diese Kopernikus-Tat, der sich Kant als solcher voll bewusst ist, ${ }^{2}$ ) vollzieht sich in dem bekannten, für das naive Bewusstsein so paradox klingenden Geêanken: Nicht schreibt die Natur „uns“ die Gesetze vor, sondern vielmehr sind „wir" es, d. h. ist es die Naturwissenschaft; welche die Naturgesetzlichkeit erst in die Wirklichkeit „hineinträgt"; ${ }^{\text {s }}$ ) die Natur als die Wirklichkeit unter der

1) Nach einem am 8. Nov. 1911 in der "Philosophischen Gesellschaft" zu Jena gehaltenen Vortrag. Für den Druck sind hinzugekommen: die Anmerkungen und eine Anzahl von Verbesserungen der Formulierung im Text, zu denen ich durch Missverständnisse, die in der an den Vortrag sich anschliessenden Diskussion zu Tage traten, veranlasst wurde. Als allgemeine Literatur zu den Gesamtausführungen ist zu vergleichen: 1. Heinrich Rickert "Geschichtsphilosophie" (in Windelbands Festschrift für Kuno Fischer „Die Philosophie im Beginn des 20. Jahrhunderts"); 2. Rudolf Eucken "Philosophie der Geschichte" (in Hinnebergs „Kultur der Gegenwart", Teil 1, Abt. 6); 3. Georg Simmel „Die Probleme der Geschichtsphilosophie", 3. Aufl., Leipzig 1907.

2) Kr.d.r. V. B Vorr. XVI.

3) Vergl. Kr.d. r. V. A 125/126. 
Form der Allgemeingesetzlichkeit ${ }^{4}$ ) ist eine Funktion der Naturwissenschaft. ${ }^{5}$ Um diese Fundamentalposition, die m. E. ein

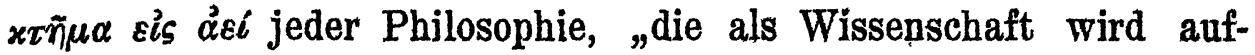
treten können", ist und bleiben wird, gleich hier in einèr modernen einwandfreieren Formulierung zu geben: Die transzendentalen Formprinzipien sind das logische Prius für die giltigen Zusammenhänge des "Gemeinten" oder "Intendierten", und zwar sowohl für den Eigenzusammenhang eines einzelnen intentionalen Gegenstandes, als auch für die Zusammenhänge der einzelnen intentionalen Gegenständlichkeiten (Sinninhalțichkeiten, Wahrheiten) unter einànder. ${ }^{6}$ )

4) Kr. d. r. V. A 263, 479.

5) Es sollte nicht notwendig sein, ist aber lẹider notwendig, ausdrücklich zu betonen, dass das Wort "Funktion" in diesen transzendentallogischen Zusammenhängen immer den aüs der Mathematik, nie den aus der Physiologie stạmmenden Siñn hat.

†) Ich will hier den Aúsdruck "transzendental", wie ich ihñ î̀ fölgenden verwenden werde, ein für allemal terminologisch festlegen: Trànszendental nenne ich jede wissenschaftliche Üntersuchung, die nicht sowohl auf eine Erkenntnis irgend welcher Gegenstände selbst abzielt, als vielmehr aúf eine Einsicht in das Wesen der "Gegenständlichkeit" von Gegenständen überhaupt. (Vergl. Kr. d. î. V. B 25). Es ist also eine Untersuchung, die nicht eine inhaltliche Gegenstandserkenntnis anstrebt, sondern bloss auf das Problem der Form (Kr.d. r. V. A 127) éines „Gegeñstand̦eș überhaupt" gerichtet ist, d. h. aự die logische "Möglichkeit" des Gegenstandes, d. h. auf den Begriff „Gegenstand“, d. h. auf den Sinn des Wortes "Gegenständlichkeit". („Beziehung auf den Gegenstand“. Vergl. Kants Problemformulierung in dem Briefe an Marcus Herz vom 21. Febr. 1772.)

Dabei ist jedoch nicht bloss an theoretische Gegenständlichkeit = Wahrheit, sonderñ auch an ästhetische, ethische, religiöse Gegenständlichkeit zuu denken, mit einem Worte: an den "Gegenstand der Kultur über-

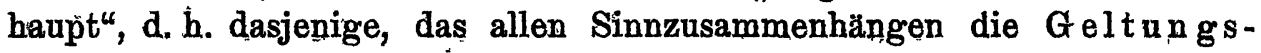
sanktion vẹrleiht; an dem sich alle Phänomene zu legitimieren haben, die Kultur im prägnanten Sinne sein wollen.

Zùsammengefasst: Transzendental ist jede Untersuchung, die (unter mêthödischer Zugrundelegung der kopernikanischen Wendung - das geht gegen jede ontologistische Metaphysik!) sich mit dem Problem der schlecht. hinigen Geltung von Prinzipien irgend welcher Sinn-, d. h. Vernunft-, d. h. Kulturzusammenbänge (wobei jede psychologisierende "Introjektion" fernzuhalten ist - das geht gegen jeden relativistischen Psychologismus!) befasst, die also auf Herausstellung von Rrinzipien der Giltigkeit schlechthin abzielt. Die Transžendentalphilosophie hat es mit dem "Logos“ zu tun, d. h. mit dem Gehalt der Kulturwirklichkeit an absoluten. Werten 'und dem Zusammenhang dieser Werte. 
Kant selbst nun stellte, der wissenschaftlichen Lage seiner Zeit gemäss, das erkenntnistheoretische Problem in Beschränkung auf eine Theorie der exakten mathematischen Naturwissenschaft, so wie er sie vorfand. Für uns heute, für die sich der Begriff der Wissenschaft nicht in dem der Naturwissenschaft erschöpft, sondern die wir (als Geschenk Hegels und der Romantik) auch eine Geschichtswissenschaft besitzen, ergibt sich aus dieser veränderten Lage der Wissenschaft im Ganzen die sowohl kulturhistorische wie pragmatische Notwendigkeit, die transzendentale Problemstellung auch auf die historische Wissenschaft auszudehnen. ${ }^{7}$ Natürlich aber ist mit dieser formalen Gleichheit oder Parallelität der Problemstellung nicht auch sehon ohne weiteres irgend eine inhaltliche Gleichheit der Lösung gesetzt, sondern diese ist, wie jede sachliche Lösung, aus der zu untersuchenden Sache selbst zu entnehmen. Mag also vielleicht - ich sage: vielleicht - die transzendentale Reflexion auf das Problem "Natur" zu dem Resultat kommen, dass die "Naturphilosophie" in einer transzendentalen Theorie der Naturwissenschaft restlos aufgehe, dass also "die Natur" überhaupt nichts anderes und nicht mehr sei, als der transzendental-logịsch konstituierte "Gegenstand" (vergl. Anm. 6) der Naturwissenschaft, so ist damit durchaus nicht etwa auch schon ausgemacht, dass pari passu das Problem

7) Trotz dieser engen Beziehung der Problemstellung einer ${ }_{n}$ Kritik der historischen Vernunft" (Dilthey) zu der in Kants Kr. d, r. V. vollzogenen "Kritik der mathematisch-naturwissenschaftlichen Vernunft" kann ich es nicht für glücklich halten, die äussere Form der Entwickelung der hier vorliegenden Probleme dem Darstellangsschema der Kr. d.r. V. anzupassen, wie es eine Abhandlung von Medicus ("Kant und Ranke" in "Kantstudien" Bd. VIII) versucht, indem sie auch bei dem historischen Bewrusstsein eine "Analytik" und eine "Dialektik" unterscheidet.

Vielmehr hätte gerade diese (natürlich nicht psychologische, sondern transzendentale) „Kritik der historischen Vernunft" an Stelle der - hẹute keinen bekämpfenswerten Gegner mehr vorfindenden - Dialektik der Kr. d.r. V. den Übergang von der reinen theoretischen Vernunft zur reinen praktischen Vernunft zu leisten. Allerdings ist eine solche Ergänzung der positiven Ergebnisse, die Kant durch eine kritische Analyse aus dem philosophischen und kulturellen Bewusstsein seiner Zeit herausschälte, eben erst durch die in der Dialektik der Kr.d.r. V. geleistete "Zermalmung" der dogmatischen Metaphysik möglich geworden: die Ausführung dieser destruktiven Arbeit durch Kant hat erst die Bahn frei gemacht für eine wissenschaftliche Weiterführung der Weltanschauungsfragen unter transzendentalem Aspekt. 
"Geschichte" in seinem Gehalt restlos konstituiert werde durch die Geschichtswissenschaft und deren Theorie. Aber soviel ist allerdings gesetzt, dass das Problem "Geschichte" von einer wissenschaftlichen Philosophie bloss angefasst werden kann durch das Problem "Geschichtswissenschaft" hindurch.

Wer also die von Kants transzendentaler Methode vollzogene kopernikanische Wendung in der philosophischen Problemstellung als zu Recht bestehend ${ }^{8}$ ) anerkennt, für den ist von vornherein klar, dass eine transzendentale Ṕhilosophie der Geschichte sich, als Ausgangspunkt wenigstens, a an die wirklich vorhandene Geschichtswissenschaft zu halten hat, unm überhaupt in ihr Problem hineinzukommen. Damit haben ,wir aber schon eine wichtige, wenn auch nur negative Einsicht für unser Problem "Geschichtsphilosophie" gewonnen, nämlich die prinzipielle Ablehnung aller Versuche, von irgend éinem der Geschichtswissenschaft selbst fremden; von aussen an sie herangebrachten Standpunkte aus die Aufgabe und Struktur der Geschichtswisssenschaft und Gesehichtsphilosophie zu bestimmen. Konkret gesprochen: Abgelehnt sind alle Versuche einer naturalistischen Geschichtsphilosophie, von dem utopischen Ideal ein er allein - seelig = machenden wissenschaftlichen Methode ausgehend, aüch die Geschichtswissenschaft und, als deren Extrakt, die Geschichtsphpilosophie in eine Gesetzeswissenŝchaft ausmünden zu lassen. Abgelehnt ist m. a. W. jeder Versuch, der Geschichtsphilosophie die șoziologische Aufgabe zu stëllen, Gesetze der sozialen Statik und Dýnamik zu suchen.

8) Iñ der Disküussion wurde betont, daș die Notwendigkeit und das Recht dieser Wendung zuerst begründet werden müsste. Das ist ohne weiteres zuzugebebn. Die Begründung hätte zu erfolgen durch eine kritische Analyse der beiden Gründbegriffspaare, in denen sich der Gedankenzusammenhang der Transzendentalphilosophie entwickelt: Form und Inhalt, Subjekt und Objekt - 2 Begriffspaare, die sich durchaus nicht decken, hat doch der eine Gegensatz seinen "Ursprung" in der "reinen" Logik, der andere in der transzendentalen Ethik im weitesten Sinne des Wortes. (Bei dem Worte „Urspring“" ist natürlich jeder Gedanke an etwas Psychologisčhes, überhaupt an irgend ein zeitlichęs Geschehen durchaus fern zu halten: es ist bloss eine Ortsbezeichnung in bezug auf das Gedankengebäude der Transzendentalphilosophie, eine Angabe, wo der betreffende Gegensatz Heimatsrecht und Unterstützungswohnsitz hat.) Aber diese Untersuchung und Bègründūng ist eine Sache für, sich; sie kann nicht an dieser Stelle und in diesem Zusammenhange in extenso gégeben, sondern muss im Wesentlichen vorausgesetzt werden. Wer hieran Anstoss nimmt, füge der Überschrift bei : „vom Standpunkte der Transzendentalphilosophie“. 
Um nicht missverstanden zu werden, will ich ausdrücklich betonen, dass auch dies vielleicht eine wissenschaftliche Aufgabe sein kann; nur ist sie nicht die Aufgabe einer Geschichtsphilosophie, die vor dem "Faktum" der einzelwissenschaftlichen Leistung, die in der wirklich vorhandenen empiriseh-positiven Geschichtswissenschaft vorliegt, so viel Respekt zu haben verpflichtet ist, dass sie von diesem Faktum, so wie es ist, als intangibler Basis ihrer Reflexionen ausgeht, statt mit mehr oder weniger Willkür der Einzelwissenschaft ins Handwerk pfuschen und ihr sagen zu wollen, was sie und wie sie eș zu behandeln habe. Die empirische Geschichtswissenschaft lehnt solch dogmatisch-spekulatives Ansinnen mit demselben Rechte $a b$, mit dem sich die exakte Naturwissenschaft gegen die dekretorischen Festsetzungen der spekulativen Naturphilosophie, etwia Schellings, gewehrt und dabei auf der ganzen Linie gesiegt hat. Seit Auguste Comte sind aber dêrartige konstruktive Versuche immer wieder aufgetaucht, und sie spielen auch noch in der Gegenwart eine Rolle; darum schien es mir nicht unwichtig und überflüssig, hier gleich in der Einleitung. dazu Stellung zu nehmen von einer ganz bestimmten, prinzipiellen Auffassung der Aufgabe und Methode der Philosophie und ihrer Stellung zu den Einzelwissenschaften aus.

Unter Geschichtsphilosophie verstehe ich also eine transzendentalphilosophische Disziplin, und deren Probleme will ich nun zu skizzieren suchen. Damit aber diese Aufgaben - denn es wird sich zeigen, dass es sịch nicht um ein, sondern um eine grosse Anzahl von Problemgruppen handelt - nicht als ein blosses, „empirisch aufgerafftes" Aggregat erscheinen, muss der Versuch gemacht werden, sie alle aus einem einheitlichen Prinzip zu "deduzieren ", sie alle auf einen Kardinalbegriff hinzuordnen, sodass ihr gegenseitiges Verhältnis und das "geistige Band“, das sie eint, zu klarem Bewusstsein kommt. Dies Deduktionsprinzip kann selbstredend nur aus dem Begriff der Transzendentalphilosophie überhaupt gewonnen werden, da deren "Wesen" sich in all ihren Ausgestaltungen und Spezialdisziplinen wiederfinden muss. Glücklicher Weise brauche ich aber im vorliegenden Gedankenzusammenhange die Wesensstruktur der Transzendentalphilosophie nicht in all ihren Einzelzügen $z \mathfrak{u}$ entwickeln, sondern es wird für unseren Zweck hier genügen, wenn ich 2 Merkmale, allerdings 2 konstituierende Grund-Merkmale, des transzendentalen modus philosophandi herausstelle, die unter sich zudem aufs engste zusammenhängen : 
1. Transzendentalphilosophie ist selbstbewusste, d. h. sich selbst wissende Wissenschaft.

2. Transzendentalphilosophie ist Geltungsphilosophie; d. h. sie hat es - im Unterschiede sowohl zu der Metaphysik alten Stiles als auch zu den positiven Einzelwissenschaften - nicht mit Fragen des Seienden, sondern mit solchen des Geltens zu tun, mit dem „Apriorischen" in der kantisierenden Terminologie, mit den Giltigkeiten und deren Prinzipien, wie wir heute lieber, unter Vermeidung des so missverständlichen und so missverstandenen Wortes "a priori“", sagen. ${ }^{9}$ )

9) Vergl. Anm. 6. Ess sei hier glèich eine weitere Ausführüung won grundlegender Bedeutung für die richtige Einstellung auf das Problem der Transzendentalphilosophie gestattet: Im Verlaufe der transzendentallogischen Untersuchüng enthüllt sich auch das Sein als ein Geltuingsproblem; darin vollendet sich erst die kọpernikanische Tat in ihrer vollen Tragweite. "Das Sein des Seienden - nicht das Seịende selber ist also eìn Spezialfall deș Gèltenden, des Reiches des Nichtwirklichen". (Hermann Leser „Einführung in die Grundprobleme der Erkenntnistheorie", Leipzig 1911, S. 250. Vergl. La's"k "Logik d. Philosophie", S. 45.) Das Sein ist eine Geltungsform neben und unter anderen Geltùngsformen.

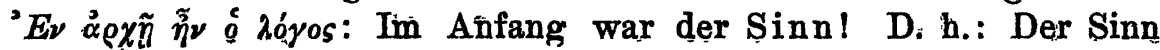
ist das logis che Prius jedes sinnvollen Zusammenhanges (also auch jedes "Wirklichkeits"-Zusämmenhanges) und damit auch jedes sinnvollen Verhaltens, z. B. des logischen Urteilens oder ethischen Handelns. Wer die logische Priorität des Sinnes vor allem - auch jẹdem idealen oder ideellen $\div$ - Sein bestreitet, behauptet damit selbstzugestandenermassen „Un-sinn". Vergl. namentlich Heinrich Rickert „Zwei Wege der Erkenntnisthèorie" (in „Kantstudien“, Bd. XIV), speziell Abschnitt V! Gerade darin, dass die Transzendentalphilosophie sich nicht von vornherein auf das. Seiende einstellt, sondern das Sein des Seienden selbst zum Problem macht, beruht ihr kritisčher, antidogmatischer Charakter. Und hat man erst einmal das Problem gesehen - darin begründet sich die Unsterblichkeit Kants! - , ist der Weg nicht mehr so weit zu der Einsicht, dass das Sein des Seienden nicht selbst wieder ein Seiu haben kann (sondern eben ñür noch einen Sinn).

Es gibt auch unter den sogenannten Philosophen eine Menge Leute, die ein der Seekrankheit ăhnliches Gefühl haben, wenn sie hören, das Sein sei eine „Kategiorie": sie glauben, den Boden unter den Füssen zu verlieren und in die Luft zu fliegen; sie haben die Vorstellung, als ob damit die Klammern, die das Weltgeftige zusammenhalten, sich in ein "aetherisches" Gebilde verflüchtigten. Wer aber die Frage nach dem Sein des Seienden, der Wirklichkeit des Wirklichen, der Gegebenheit des Gegebenen usw. nicht von den Fragen nach dem Seienden, Wirklichen, Gegebenen usw. 
Das erste Moment ist unschwer klar zu machen. Die Einzelwissenschaften untersuchen und prüfen nicht selbst die letzten, obersten Prinzipien, von denen sie bei ihrer Arbeit ausgehen, bezw. gemäss denen sie in ihrer Arbeit verfahren. Aus dieser Sachlage entspringt ja gerade das Bedürfnis nach einer eigenen Disziplin, die das tut: die Notwendigkeit einer Erkenntnistheorie als eigener Wissenschaft mit eigenem Gegenstand. Anders in der Philosophie: da es über sie hinaus keine Wissenschaft mehr gibt, die ihre Prinzipien untersuchen könnte, muss sie das prinzipiell selbst tun, gerade wenn und weil sie die letzte und oberste Wissenschaft zu sein beansprucht. Das heisst: sie hat jeden einzelnen Schritt, den sie tut, mit vollem Bewusștsein seiner Gründe und seiner Trag-

selbst zu trennen vermag, ist eben in logischer Hinsicht formenblind: er hat kein Recht, die transzendentale Problemstellung weder anzunehmen noch abzulehnen, da er sie noch gạr nicht sieht; der philosophische Star ist ihm noch nicht gestochen, ihm fehlt das transzendentale Sehen.

Man hat von der Infinitesimalrechnung gesagt, sie sei die Wissenschaft, "welche das Gras wachsen hört" (vergḷ. Liebmann "Gèdanken und Tatsachen" II, 103 Anm.): ein ausgezeichnetes Bild! Eines von den wenigen Bildern, die wirklich geeignet sind, auch einem im rein logischen Denken weniger geschulten Kopfe ein rein begriffliches Verhältnis anschaulich näher zu bringen. Aber es gilt viel allgemeiner: von der gesamten Transzendentalphilosophie. (Cohen.) Die transzendentale Problemstellung sieht letzten Nndes auch in jeder Tat- ${ }_{n}$ sache", in jeder "Wahr"-nehmung ihr Konstituiertsein aus transzendentalen Formprinzipien, aus denen allein sie ihre "Geltung" entnimmt, ihr transzendentales "Erzeugt" ${ }^{\text {-sein aus ihren }}$ logischen „Möglichkeitsbedingungen".

Aber wohlgemerkt: Jeder Gedanke an eine "erzeugende Tätigkeit", einen Akt des Subjekts ist in der "reinen Logik" durchaus fern zu halten. (Vergl. das Wort "Ursprung " in Anm. 8.) Die Notwendigkeit der kopernikanischen Wendung entspringt in der transzendentalen $\mathrm{L}$ o gik und betrifft zunächst bloss den Gegensatz von Form und Inhalt, nicht den von Subjekt und Objekt. (Vergl. darüber Emil Lask "Die Logik der Philosophie und die Kategorienlehre" [Tübingen 1911].) Erst in der transzendentalen Ethik wird das Subjekt von zentraler Bedeutung. „Im Anfang war der Sinn": von diesem zeitlos giltigen Sinn handelt die transzendentale Logik. Erst wenn es sich dann um die "Verwirklichung" einer Giltigkeit handelt, ihre Herabholung aus der sich selbst genügenden Sphäre des reinen Geltens in die der physisch-psychischen Wirklichkeit, ihre Utberführung in physisch-psychisch Wirklich es, wird aus jenem ersten „Grundsatze" die zweite "Grund-setzang": „Im Anfang war die Tat". Handelt die transzendentale Logik vom zeitlos giltigen theoretischen Sinn, so handelt die transzendentale Ethik von der zeitlos giltigen praktischen Tat (Handlung, „Tathandlung ${ }^{a}$ ). 
weite, seines Sinnes und seiner Geltung ${ }^{10}$ ) zu unternehmen. Wenn aber so die Transzendentalphilosophie selbstbewusste, d. h. sich selbst wissende Wissenschaft sein muss, so folgt daraus, dass jede Einzeldisziplin derselben nicht nur Philosophie ihres Gegenstandes, also etwa der Natur oder der Geschichte, zu sein hat sọdern immer auch und zuerst - nicht nur Philosophie der Wissenschaft von diesem Gegenstande, sondern - auch Philosophie der Philosophie dieses Gegenstandes. Auf die eminent schwierigen logischen Probleme, die sich hier erheben, ${ }^{11}$ ) kann ich an dieser Stelle nicht eingehë̈; ich will aber en passant eine in anderer Richtung liegende Konsequenz dieses Charakters der Philosophie ausdrück= lich ins Bewusstsein heben: ${ }^{12}$ ) sie bezieht sich, wenn ieh es so nennen darf, auf die "Ethik des Philosophierens".

Wenn nämlich die Philosophie allübẻrâll sìch selbst wissend̄es Wissen ist, bezw. seini soll, so ergibt sich als "kategorischen Imperativ" für die Denkarbeit des Philosophen, will sie Wissenschaft schaffen, dass er ñiemal̆s (um den Ausdruck Schillers zu verwënden) als "schöne Seele" philosophierèn darf́, sondern - als "Moralist des Denkènsं“ 18) - immer zu vollem kritischem Bewusstsein seiner Grundsätze .verpflichtet ist bei jedem eínželnen Denkakt, sofern er für deñselbeñ uñd dessen Ergebnis Allgemeingiltig-

10) Auch seineṛ "Gẹltung": darin kommt, wie oben gleich betont, der enge Zusammenhảng dieses erșten Merkmals der Tranșendeñtalphilosophie, ihrer Sèlbstbewैusstheit, mit dem nachher zu behandelnden zweiten, ihrem. Charakter als Geltungsphilosophie, zum Vorscheín. Diese enge Beziehung ist nicht weiter verwunderlich, wenn mañ eingesehen hat, dass der eigentliche Kern jedes "Selbstbewusstseins" das "Geltungsbewusstsein "ist. "Selbstbewusstsein " ist das Bewusstsein des einheitlichen Geltunğszüsan̄menhangs aller Eigenerlebnisse in dem Subjekts-grundw̄ert "Ich". Was dieses Selbstbewusstsein eines Subjekts, die Einheitsform "Ich", den empirisch-individuell-giltigen Erlêbnissèn leistet, das will die Transzendentalphilosophiẹ der Gesamtheit der überempirisch-überindividuellen Giltigkeiten schlèchthin leistěn. Und umgekehrt ist gerade das Selbstbewusstsein dasjenige Moment an dem individuellen Sụbjekt, durch welches dieses in die transzendentale, d. h. reine Geltungssphäre hinaufragt.

11) Ich verweise hierfür auf das in Anm. 9 zitierte Buch von Lask, in dem zuerst in prinzipiell durchgeführter Weise über der Logik der 4 Seiniswissenschaften (Natur= und Geschichtswissenschaft) eine Logik der "Geltungswíssenschaft, d: i. der Philosophie verlangt wird. führe.)

12) (in der ich eine Anregung meines Freundes Otto Baensch weiter-

13). Ottó Liebmann: „Zur Analysis der Wirklịchkeit“, S. 546. 
keit beansprucht. Für ihn als Philosophen und sofern er philosophiert, gilt in der Tat, was Kants "Maximenmoral" für das gesamte menschliche Handeln, sofern es sittlich sein will, verlangen zu müssen glaubte - gegen welche lebentötende Überspannung des Rationalismus man sich mit Recht aufgebäumt hat. Von dem Philosophen als solchem muss die Wissenschaft schlechterdings überall und immer zwar nicht den Rationalismus, wobl aber die durchgängige bewusste Herrschaft der ratio, d.h. des methodisch-wissenschaftlichen Begründungszusammenhangs verlangen. Konkreter gesprochen: Alles sogenannte "genialische" Philosophieren ist so lange vor-wissenschaftlich, und zwar im Sinne von unter-, nicht von überwissenschaftlich, solange es nicht seine „Schauungen“ und sein „Geschautes" aus dem "Aggregatzustand" der intuitio, in dem es konzipiert sein mag, in den der ratio, d. $h$. der in diskursiver Form sich entwickelnden Vernunft als wissenschaftliches System übergeführt hat. Die Philosophie als Wissen-

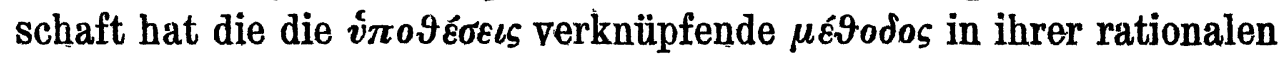
Konstitution ins Bewusstsein zu heben.

Transzendentalphilosophie ist selbstbewusste, $d . h$. sich selbst wissende Wissenschaft. Daraus rechtfertigt sich, dass man, bevor man die einzelnen sachlichen Probleme der Geschichtsphilosophie zu behandeln unternimmt, zuerst das Problem "Geschichtsphilo-

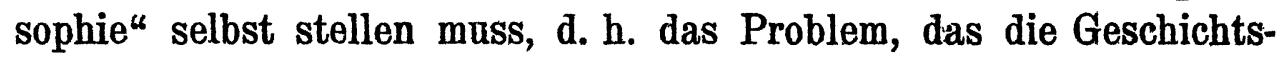
philosophie als transzendentalphilosophische Disziplin der auf sich selbst reflektierenden Philosophie stellt; Kantisch ausgedrückt: die Frage nach der „Möglichkeit der Geschichtsphilosophie“, d. h. nach ihrem Sinn, ihrem Begriff, ihrem Wesen als philosophischer Wissenschaft, und dem daraus sich ergebenden Zusammenhang ihrer Probleme (wozu eben dieser Aufsatz eine Studie sein will).

Was nun das zweite Merkmal anlangt: Transzendentalphilosophie ist Geltungsphilosophie, so muss man hier, will man verständlich sein, entweder ganz weit ausholen oder sich ganz kurz fassen; ich wähle zeit- resp. raumgedrungen das letztere. Es empfiehlt sich vielleicht als das Einfachste, von einer allbekannten Behauptung Kants selbst auszugehen: In der Transzendentalphilosophie handele es sich nicht um quaestiones facti, sondern um quaestiones iuris. ${ }^{14}$ ) Die Untersuchung der ersteren, wie sie die Einzelwissen-

14) Vergl. den philosophisch zentralen Abschnitt der „Kr.d.r. V." B 116 ff. ( $n$ Von der Deduktion ${ }^{\prime}$ usw.). 
schaften vornehmen, kann zwar zur Kenntnis des kritisch zu Beurteilenden beitragen; niemals aber kann sie aus sich selbst heraus, gerade weil sie sich prinzipiell nur mit Urteilen über das Seiende und dessen Genese befasst, Kriterien für dessen Beurteilung liefern. Auch diese aber sind ein würdiger Gegenstand menschlichen Wissens; anf sie hat sich im Unterschiede von den die Seinsprobleme behandelnden positiven Wissenschaften die Philosophie zu richten. Der logisch übergeordnete Begriff für quaestio iuris = Rechtsfrage aber ist Wertfrage, und darum hat Windelband ${ }^{15}$ ) die Transzendentalphilosophie nach Gegenstand und Methode als "kritische Wertwissenschaft" definiert. Die neueren logischen Untersuchungen haben hier weiter gebohrt und gezeigt, dass auch der Wertbegriff hier noch nicht der logisch letzte sei, sondern dass der rein-logische Gehalt des Wertbegriffs sich in dem Lôtzeschen Begriff des Geltens erschöpfe. ${ }^{16}$ ) So wird-der Begriff des Geltens zum Kardinalbegriff der modernen Transzendentalphilosophie als Wissenschaft.

Aus ihm resultiert erst der Begriff des Wertes durch inhaltliche Eigentümlichkeiten des dem Gelten (in der erkenntnistheoretischen Abstraktion) gegenüberstehenden Seienden als dem „blossen Materiale" (Fichte!) für das Gelten, nämlich daraus, dass es darin Subjekte gibt: der Begriff des Geltens wird zu dem des Wèrtes erst durëh die Subjektbezogenheit, d. h. dadurch, dass das Gelten nicht in seiner logischen Reinheit als Gelten schlechthin ins Auge gefasst, sondern auf ein Subjekt, bezw. ein Subjekt auf es beżogen wird, für das es gilt: Wert $=$ sübjektbezogenes Gelten.

Wo es sich also in der Philosophie um ein Gebiet handelt, bei dem von dieser Subjektbezogenheit des Geltens abstrahiert

15) Vergl. den grundlegenden Aufsatz seiner „Praeludien": „Was ist Philosophie?"; ferner ebendaselbst "Kritische oder genetische Methode"; endlich im „Logos“, Bd. I, S. 188 ff.: „Kulturphilosophie und transzendentaler Idealismus".

16) Hèrmànn Lotze: "Lògik", Buch III, Kap. 2 "Die Ideenwelt". Vergl. namentlich S. 500/501 (zit. nach der 1. Aufl.): „Was dieses Gelten heisse, muss man nicht wieder mit der Voraussetzung fragen, als liesse sich das, was dạmit verständlich gemeint ist, noch von etwas Anderem ableiten". "Man muss auch diesen Begriff als einen durchaus nur auf sich beruhenden Grüdbegriff ansehen,, von dem jeder wissen kann, was er mit ihm meint, den wir aber nicht durch eine Konstruktion aus Bestandteilen erzeugen können, welche ihn selbst nicht bereits enthielten". 
werden kann oder muss, wie z. B. in der transzendentalen Theorie der exakten mathematischen Naturwissenschaft, da tritt der Wertbegriff zurück, und alles dreht sich um den Begriff der Giltigkeit und deren Zusammenhangsprinzipien. Und zwar sind diese Giltigkeiten durchaus keine mysteriösen Gebilde, sondern die uns allen bekannten "Naturdinge" als "Gegenstände der Naturwissenschaft", bezw. die naturwissenschaftlichen Begriffe oder Gesetze, die nur zum Zwecke einer logischen Theorie als eines Teiles der Transzendentalphilosophie überhaupt die Bezeichnung "Giltigkeit" bekommen, weil in ihrem schlechthinigen theoretischen Gelten ihr „Wesen" als Gegenstände der Naturwissenschaft sich restlos erschöpft.

Diese Betrachtung unter dem Gesichtspunkte des reinen Geltens verschiebt sich aber sofort mit dem Momente, wo ein Menșch nicht als blosses $\mathrm{Objekt}$ der Naturtheorie, sondern als wollendes und handelndes Subjekt in den Gesichtskreis der. transzendentalen Reflexion tritt. An Stelle der Probleme des reinen Geltens erhebt sich nuu das Problem des Geltens inbezug auf ein handelndes Subjekt, d. h. das Problem des Wertes. Der Begriff des Wertes überhaupt aber zerlegt sich dann weiter durch seine Beziehung auf das Subjekt des Handelns einerseits, das Handeln des Subjekts andererseits wieder in zwei Begriffe: 1. als das Gelten für noch $\mathrm{zu}$ geschehende Handlungen ergibt sich der Begriff der Norm, 2. als das Gelten von schon geschehenen Handlungen ergibt sich der Begriff des Sinnes. Ist jene der formale Grundbegriff der Ethik, so ist dieser derjenige der Geschichtsphilosophie.

Die gesuchte Deduktion der Aufgabe der Geschichtsphilosophie innerhalb der Gesamtaufgabe der Transzendentalphilosophie als sich selbst wissender Geltungsphilosophie ergibt das Resultat: sie handelt nicht von Gesetzen des Geschehens, sondern ist das wissenschaftliche Selbst-Bewusstsein vom Sinn der Geschichte.

Da aber „der Sinn sich nur deuten lässt auf Grund von Werten, die gelten ${ }^{4},{ }^{17}$ ) gründet die Geschichtssinnwissenschaft in der Geschichtswertwissenschaft. Von diesem Begriff und den in ihm gesetzten Problemen müssen wir ausgehen, um die Gesamtheit geschichtsphilosophischer Probleme in ihrem systematischen Zusammenhang zu überblicken.

17) Heinrich Rickert „Lebenswerte und Kulturwerte ${ }^{\alpha}$, im "Logos" II, 
Wie das Wort "Episode" nicht nur den eingelegten Gesang, die $\dot{\epsilon} \pi \varepsilon \iota s \omega \delta \eta^{\prime}$, bezeichnet, sondern auch, ja in erster Linie das darin Besungene, das eingeschobene wirkliche Ereignis, so hat auch das Wort "Geschichte" die Doppelbedeutung, sowohl den realen historischen Prózess zu bedeuten, als die Wissenschaft von demselben. Dieser Zweiteilung entsprecehend ist darum auch die Geschichtsphilosophie sowohl Wissenschaft vom Werte (oder Sinn) der Geschichtswissenschaft, als auch Wissenschaft vom Werte (oder Sinn) der Geschichte selbst. Gemäss früheren Ausfiuhbrungen kann sie als transzendentale Disziplin nur durch das Problem der Geschichtswissenschaft hindurch an das Problem der Geschichte selbst in wissenschaftlich-philosophischer Weise herankommen.

Wir erhalten also zunächst zwei grosse Gruppen geschichtsphilosophischèr Probleme: Geschichtswissenschaftswertwissenschaft und Geschichtswirklichkeitswertwissen. schafit. Jeder dieser Problemkomplexe zerlegt sich aber sếinerseits wieder in zwei Problemgruppen gemäss folgendẹr Überlegung:

1. Wenn ich vom Werte der Geschichtswissenscháft hañdèle, so kañn ich einmal dieșe als Gesamtheit hinnehmen und nach ịhrem Wert für ausserhalb ibrer selbst liegende Werte fragen, also vor ällèm nảch dem Wert der Geschiehtswisseñschaft für das Leben einerseits, für Philosophie und Weltanschaúung andererseits. Diese Probleme wollen wir als Geschichtswissens.chaftswertwissenschaft im engeren Sinne zusammenfassen.

Ich kann aber auch die Geschichtswissenschaft für sich als einen Eigenwert, eben den Wert „Geschichtswissenschaft" (um der Geschichtswisisšenschaft willen) ins Auge fassen und hier nun innerhalb dieseș Wertes nach den Prinzipien fragen, die ihn als Wert konstituieren, d. h. also nach der Struktur der Geschichte als Wissenschaft, nach deren Voraussetzungen und Methode. Diese Problemgxuppe wollen wir bezzeichnen als kritische Geschichts̃wissenschaftswissens chaft.

Das wäre also die Philosophie der Geschichtswissenschaft. Die Aufgàben der Philosophie der Geschichte sélbst aber erwachsen hieraus auf Grund folgenden Weiterdenkens:

2. "Nicht älles, was geschieht, ist Geschichte" (Windelband). Die Geschichtswissenschaft bedarf also Prinzipien, nach denen sie aus der Männigfaltigkeit der Geschehnisse ihre Auswahl trifft und gemäss denen sie ihre historischen „Begriffsbildungen". heraus= 
stellt. Diese „Prinzipien der Synthesis" sind aber eben als solche Wertprinzipien, aus denen erst der betreffenden " Ordnung" von Tatsachen der Charakter als Geltungszusammenhang zufliesst. Der empirische Historiker braucht sich dieser seiner transzendentalen Bedingtheit natïrlich ebenso wenig bewusst zu sein bei der Einzelarbeit innerhalb seiner Wissenschaft, als der Naturwissenschaftler auf seinem Arbeitsgebiete. Der Geschichtsphilosophie aber erwächst die Pflicht, nachdem sie diese transzendentalen Bedingungen in der Geschichtswissenschaftswissenschaft bloss auf ihre Funktion als Wissenschaftskonstituens untersucht hat, sie nun weiter, ihre transzendentale Analyse vertiefend, auch als Gegenständlichkeitsprinzipien der Geschichte als realem Prozess ins Auge zu fassen. - Das heisst: sie hat den transzendentalen Gehalt der Geschichte selbst zu untersuchen und herauszustelleñ.

Diese Aufgabe aber zerlegt sich in eine materiale und eine formale: es sind zunächst die inhaltlichen historischen Werte, die die empirische Geschichtswissenschaft herausarbeitet, auf ihren (nicht historischen Seins-, sondern überhistorischen) Wertzusammenhang unter einander zu untersuchen, und es muss dann zweitens der letzte Grund und Sinn all dieser Werte und ihres Zusammenhangs, also das' unterste Fundament der Geltung von Werten überhaupt, geprüft werden. So ergeben sich innerhalb der Geschichtswirklichkeitswertwissenschaft auch wieder zwei Problem-. gruppen: kritische Wissenschaft von den historischen Werten und kritische Wissenschaft von dem Geltungsgrund dieser Werte; mit analoger Wortbildung wie bei 1: kritische Geschichtswertewissenschaft und kritische Geschichtswertewertwissenschaft.

Diese Wortbildungen klingen, wie ich selbst sehr wohl weiss, alle nicht sehr schön. Aber sie haben den Vorzug, das gemeinsame Band, das all diese in sich sehr verschiedenen Probleme unter einander zusammenhängen lässt, schon äusserlich hervortreten zu lassen. Ich werde selbst nachher für die Grundprobleme andere Bezeichungen einführen, will aber als Zusammenfassung meiner bisherigen Ausführungen die 4 Disziplinen, in die sich uns „das Problem der Geschichtsphilosophie" nunmehr zerlegt hat, nochmals hinter einander aufzählen:

Die Geschichtsphilosophie als transzendentale, d. h. wertkritische Geschichtssinnwissenschaft zerfällt in: 
I. die Geschichtswissenschaftswertwissenschaft und II. die Geschichtswirklichkeitswertwissenschaft. Erstere wieder zerfallt in:

1. die Geschichtswissenschaftswert-wissenschaft i. e. S. und 2. die kritische Geschichtswissenschafts-wissenschaft. Letztere zerfällt in:

3. die kritische Geschichtswerte-wissenschaft und 4. die Geschichtswertèwert-wissenschaft.

Der allgemeine Teil ist damit żu Ende. Ich will in eỉnem 2. Teile versuchen, die so in abstrakto unterschiedenen Problemgruppen einzeln etwas näher zu beleuchten; zu grösserer Veranschaulichung und Verlebendigung werde ich dabei einige"gegenwartsphilosophiegeschichtliche Ḧinweise zu geben suchen, indèm ich einige markante Strömungen, Hauptprobleme, Denker, Bücher in meine. Problemgruppierung einreihe.

Bei der ersten Problemgruppe kann ich mich kurz fasssen. Die Fragen, um die es sich hier handelt, sind keine gesçhichtsphilosophischen im eigentlichen Sinne, sondern mussten hier nur mit angeführt werden, weil sie zu diesen in enger Beziehung. stehen, hầufig auch als "Geschichtsphilosophịe" bezeichnet werden, und in der Tat nur aus dieser heraus eine begründete Antwort finden können.

Dás Problem: "Geschichtswïissenschaft und Lebeñ " ist ein pädag ogis ches Problem, Pädagogik in dem umfassenden Sinne einer Bildungswisissenschaft überhaupt genommen: es handelt sich um die Frage nach dem "Wert der historischen Bildung“. Gegen Nietzsches „unzeitg̈èmässe (!) Betrachtung“: „Vom Nachteil der Historie für das Leben". möchte ich die schönen Worte eines Mannes anführen, der von schulphilosophischer Beschränktheit mindestẹns so frei war, wie Nietzsche, und doch auch was von der Sache verstand, W. v. Humboldts: ${ }^{18}$ ) ;Die Geschichte dient nicht sowohl durch einzelne Beispiele des zu Befolgenden oder Verhütenden, die oft irreführen und selten belehren. Ihr wahrer und unermesslìcher Nutzen ist es, mehr durch dié Form, die an den Begebenheiten hängt, als durch sie selbst, den Sinn für die Behandlüng der Wirklichkeit $\mathrm{zu}$ beleben und $\mathrm{zu}$ läutern, zu verhindern, dass er nicht in das Gebiet blosser Ideen überschweife, und

18) „Ausgewählte philos. Schriften" (Phîl. Bibl. Bd. 123), S. 84. 
ihn doch durch Ideen zu regieren, auf dieser schmalen Mittelbahn aber dem Gemüt gegenwärtig zu erhalten, dass es kein anderes erfolgreiches Eingreifen in den Drang der Begebenheiten gibt, als mit hellem Blick das Wahre in der jedesmal herrschenden Ideenrichtung zu erkennen, und sich mit festem Sinn daran anzuschliessen."

Im Allgemeinen ist zu sagen: dass zwar einerseits ein über den Positivismus und Relativismus der empirischen Geschichtswissenschaft nicht hinauskommender Hist oris mus ebenso einseitig und unzulänglich ist, als ein die Geschichtswissenschaft einfach ablehnender Naturalismus, dass aber andererseits gerade dieser letztere absolut ungeeignet ist, zu jener Einseitigkeit die Ergänzung zu geben, sondern dass diese nur aus einer in absoluten Werten sich gründenden Kulturphilosophie als Wissenschaft fliessen kann. Denn „der Kulturmensch ist überhaupt nicht schon mit und in dem natürlichen Menschen gegeben, sondern für den geschichtlichen Menschen aufgegeben und wird durch inn allein verwirklicht. Darum besteht ja alle Erziehung, die wir leisten, alle Bildung, die wir leiten können, wesentlich darin, aus dem natürlichen Menschen den historischen zu machen. " ${ }^{19}$ ) Nur von diesser allgemeinen Position aus kann dann auch die speziellere Frage entschieden werden, ob unsere Schulbildung humanistisches oder realistisches Grundgepräge tragen soll. Für die Selbsterziehung aber fliesst aus der Versenkung in die Geschichte die Erarbeitung „eines Lebensstandpunktes, der über die Einseitigkeiten der eigenen Natur hinweg ist und in reiner Klarheit des Gedankens vor sich liegen sieht, was andere als dunkle Macht beherrscht". ${ }^{20}$ )

Die andere Frage in dieser ersten Problemgruppe ist die nach dem Verhältnis der Geschichtswissenschaft zu Philosophie und Weltanschauung, zunächst in rein formaler, methodologischer Hinsicht. Damit ist das m. E. schwerste Problem der Transzendentalphilosophie als Wissenschaft ïberhaupt aufgeworfen, das grundlegende Methodenproblem, die Frage nach dem "Organon “ ${ }^{21}$ ) der Geltungsphilosophie. Ich kann hier natürlich bloss die Möglichkeiten, so wie ich sie sehe, kurz skizzieren. ${ }^{21}$ ) Seite 10.

19) Windelband "Wesen und Wert der Tradition im Kulturleben",

20) Ed. Spranger im Nachruf für Dilthey im "Zeitgeist".

21) Vergl. Kants "Kr. d. r. V." B, S. 24/25.

21 a) Vergl. zu dem zunächst Folgenden: Windelband „Die Erneuerung des Hegelianismus". 
Es handelt sich zunächst um die beiden - auch gerade in der Gegenwart wieder lebendigen - Antipoden: Fries und Hegel, d. h. um die Frage, ob die Psychologie oder die Geschichte die Fundstätte der kritischen Erarbeitung der transzendentalen Giltigkeiten sei; beide Methoden, die psycho-kritische und die historio-kritische, bilden schon die antithetischen Pole in der Entwickelung Kants selbst von der „psychologischen Erkenntnistheorie der Inauguraldissertation" bis zur rein kritischen Fragestellung der "Religion innerhalb der Grenzen der blossen Vernunft".

Wir selbst haben schon soeben in der Unterscbeidung des "natürlich gegebenen" animal soziale und des "historisch aufgegebenen" Vernunftmenschen Stellung genommen: nicht durch psychologische Untersuchungen, sondern „aus dem historischen Kosmoś, wie ihn die Erfahrung der Kulturwisșenschaften darbietet, sind die Prinzipien der Vernunft herauszuarbeiten" (Windelband, loc. cit., S. 11). Oder, wie Rickert es formuliert: „Nur durch das Historische hindurch känn der Weg żum Übërhistọischen führen. An dem historischen Material hat die Philosophie die Werte als Werte sich zuum Bewusstsein zu bringen. "22) [Darin begründet sich dann weiter, was hier nicht näher ausgeführt werden kannn, die eigentümliche, einžigartige Stelliung, in der die "Geschichte der Philosophie" zur systematischen Philosophie steht].

Aber natürlich, ist mit diesem allgemeinen, transzendentalmethodologischen Bekenntnis zu Hegel gegen Fries zunächst nư negativ die Ablehnung der Psychologie als Grundlage der Transzendentalphilosophie ausgesprochen, das positive Verhältniss zur Gieschíchtè selbšt lässt zunächst verschiedene methodische Möglichkeiten offen. Ja, auch jẹne Ablehnung der Psychologie umfasst nicht die Ablehnung der "Phänomenologie", wie sie Husserl in seinen "Logischen Untersuchungen" betreibt, ${ }^{28}$ ) und nicht die der "beschreibenden und zergliedernden Psychologie“ Dilthey's, die vielmehr beide unter geltungsphilosophischem Aspect und in transzendentalen Hilfsdiensten stehen, wenn sie es auch beide nicht Wैort haben wैolleñ.

22) Rickert: „Vom Begriff der Philosophie", im „Logos", Bd. I, S. 18.

23) Wohl aber des allgemeinen Programms, das er daraus in dem Aufsatz "Philosophie als strenge Wissenschaft" („Logos“ Bd. I. S. 289 ff.) entwickelt. 
Was nun die historiokritische Problemstellung betrifft, so ist damit keineswegs auch schon dje „dialektische Methode" Hegels in Bausch und Bogen rezipiert. Es bieten sich hier im wesentlichen drei prinzipielle Mögliehkeiten, indem drei verschiedene methodische Ansatzpunkte für eine philosophische Bearbeitung von Geschichte und Kultur vorliegen, wobei der Begriff der Kultur mit Bauch so zu definieren ist: ${ }^{24}$ ) „Ist die Natur das Dasein, sofern es nach allgemeinen Gesetzen bloss mechanisch bestimmt ist, so ist die Kultur das Dasein, sofern es nach allgemeinen Gesetzen zweckvoll, d. h. kurz: nach Zwecken bestimmt ist." Um nun das "Wesen" der Kultur żù erfassen, kañn mán 1. so vorgehen, dass man sieh an den realen historischen Prozess in seiner Bewegung hält, und in diesem die Kräfte zu fassen súcht, die das Herauskommen der Kultur bewirken. Dies dürfte der Weg sein, auf dem Eucken zu einer überhistorischen Kulturphilosophie zu gelangen sucht. Oder aber man versucht 2. die transzendentalen Prinzipien aus den sich selbst genügenden Niederschlägen des Kulturprozesses, den historisch vorliegenden Kulturresultaten zu gewinnen; also etwa bei dem Kulturgebiet „Wissenschaft": aus den "gedruckt vorliegenden Büchern" als Kulturdọkumenten. Das ist die Philosophie der "Marburger Schule*. Oder endlich 3. man sucht von der Idee der Kultur aus ihre Wertkonstituentien zu erfassen, wobei aber diese Idee nicht als konstruktives Ideal, sondern als transzendentale Möglichkeitsbedingung gemeint ist. Diese 3. Methode zu einer transzendentalen Kulturphilosophie als Wissenschaft ist diejenige Windelbands und Rickerts.

Soll ich für die 3 Richtungen philosophie-geschichtliche Anlehnungspunkte (in rein sachlicher Hinsicht) angeben, so würde ich die erstere der Kr.d.pr. V., die zweite der Kr.d.r. V., die dritte der Kr. d. U. zuordnen, oder auch die erstere mehr auf Fichte, die zweite auf Kant, die dritte auf Hegel hinorientieren.

Was meine eigene Stellung angeht, so würde ich gegen Eucken betonen, dass für diese Philosophierweise die Gefahr eines "metaphysischen Dogmatismus" sehr gross ist. Bei aller Anerkennung and Wertschätzung des ethischen Pathos, von dem die Gesamtanschauung getragen ist, muss gesagt werden: logisch nicht

24) Bauch "Über den Begriff der Geisteskultur" (in "Religion und Geisteskultur", Bd. I, S. 153. 
geschulte Köpfe können leicht dazu kommen, den logischen Geltungssinn und Geltungsgehalt dieser Ausführungẹn nicht richtig einzuschätzen und als bewiesene Resultate einer wissenschaftlichen Philosophie zu besitzen glauben, was nur intuitiv aufgestellte Kulturpostulate und daraus gesetzte Überzeugungen über das Wesen der Welt sind, deren Geltung (als „Wahr") selbst erst eine - erkenntnistheoretisch zu fandierende - transzendentale Werttheorie voraussetzt. In der 3. Richtung scheinen mir die berechtigten Momente der Position Euckens „anfgeboben“ zu sein.

Der 2. Richtung aber ist die 3. dadurch überlegen, dass sie sich von vornherein einstellt auf den "Wertbegriff der Philosophie als "teleologia rationis humanae", wie ihn Kant in der "Methoden= lehre" der Kr. d. r. V. ${ }^{25}$ ) aufstellt. Innerhalb dieser ist die Wissenschaft, in specie die mathematische Naturwwissenschaft, nur ein Kulturgut neben anderen, unnter anderen. Darum darf die auf sie passende und an ihr bewährte Methode nicht öhne weiteres zu der Methode gemacht werden. Eș ist falsch, dass die Philosophie nưr durch die Wisseñschaft, nicht ãnders̀, sich auf das Ganze der Kultur bezieht. ${ }^{26}$ ) Auf diese Weise ist weder dem ethischen, noch dem ästhetișchen, noch dem religiösen Problem adäquat beiżükommen (trotzdem natürlich deren philosophișç̣e Behandlung als solche Wissenschaft sein muss). V̌on hier aus gestaltet sich abèr dànn der ganze Systemzusammenhang anders als bei Cohen und Natorp. Auch die in dieser Richtung berechtigten logischen Momente scheinen mir in der "kritischen Geltungsphilosophie" „aufgehoben" zu sein. ${ }^{27}$ )

Ich verlasse die grundlegende, methodologische Vorfrage und gehe zum 2. Problem: der Geschichtswissenschaftswissenschaft über.

${ }^{25)}$ „Kr. d. r. V." B., S. 867 ff.

26) Natorp: „Philosóphie“, Göttingen 1911, S. 26/27. Entsprechend legt Cohen, wie der Logik die math. Naturw., der Ethik die Rechtswissenschaft zu Grunde.

27) Da in dem von Rickert und seinen engeren Schülern (z. B. Hessen) bevorżugten Namen "trianszendentaler Empirismus" (im Gegensatz zum "transzendentalen Rátionalismus" der Marburger) die Beziehưng auf Geschichte und Kultur nicht zum Ausdruck kommt, möchte ich die Bezeichnung "transzendentaler Historiokritizismus" vorschlagen, (wenn man nicht einfach, im Gegensatz zum "transzendentalen Naturalismus" der Marbuirger - vergl. "Grosse Denker" hergg. von v. Aster, Bd. II, S. 371 -, diese auf den Logos der Geschichte sich gründende Weltanschaunng "transzendentalen Culturalismus" nennen will). 
Ich knüpfe an das an, was ich oben über den Charakter der Selbstbewusstheit aller transzendentalphilosophischen Untersuchungen ausgeführt habe. Die Anfangsgründe jeder einzelnen Spezialdisziplin der Transzendentalphilosophie sind danach nicht Philosophie ihres Gegenstandes, sondern Philosophie der Philosophie dieses Gegenstandes, stellen also ein Stück Philosophiephilosophie dar. Ist aber nun dieser Gegenstand selbst eine Wissenschaft, so ist der sich ergebende Tatbestand folgender: Jede Einzelwissenschaft hat ein Bewusstsēin von ihren Gegenständen, ja, sie ist dies "Bewusstsein von " ihren Gegenständen; denn jede Wissenschaft als ein eigengründiger Zusammenhang giltiger Sätze ist transzendentallogisch nichts anderes; als eben der geltende Zusammenhang ihrer Gegenstände, oder, von der Seite des Subjekts gesehen, nichts anderes, als das betreffende Stück oder die betreffende Seite des Wirklichen in der gerade durch den jeweils vorliegenden wissenschaftlichen Auswahl- und Anordnungsgesichtspunkt bedingten synthetischen Einheitsform. Wegen der durchgängig bestehenden transzendentalen Funktionalgleichung zwischen Giltigkeit und Gegenstand sind das ja eben bloss zwei Seiten einer Gleichung, also zwei verschiedene Ausdrücke für dieselbe Sache. Jede Eịnzelwissenschaft ist also die Gesamtheit ihrer Gegenstände in der Einheitsform ihres Bewusstseins.

Aber von diesem ihrem Bewusstsein und dessen synthetischen Strukturformen nach ihrem Sinn und ihrer Geltung hat sie als Einzelwissenschaft nicht selbst wieder ein Bewusstsein. Sondern dies Bewusstsein von 'ihrem Bewusstsein, ihr (d. h. der Einzelwissenschaft) Selbstbewusstsein hat die Philosophie, ișt Philosophie. Daraus aber ergibt sich, wiederum wegen der kategorial-logischen Konstituiertheit jedes Gegenstandes irgend einer Wissenschaft durch die transzendentalen Prinzipien, denen sie als Wissenschaft untersteht, dass die letzten Fundamente jeder Wissenschaft als Wissenschaft nichts anderes sind als ihr transzendentaler Gehalt. Etwas prononzierter ausgedrückt: Das eigentlich Wissenschaftkonstituierende an jeder Wissenschaft ist philosophischer Natur; die Einzelwissenschaften enthalten genau so viel eigentliche Wissenschaft, als sie Transzendentalphilosophie enthalten.

Die Selbstüberhebung der Philosophie, die hierin zu liegen scheint, verschwindet sofort, wenn man sich klar macht, 1. dass die Transzendentalphilosophie nicht identisch ist mit dem, was dieser oder jener Transzendentalphilosoph meint; ebensowenig wie 
sich die Naturwissenschaft deckt mit dem, was irgend ein Naturwissenschaftler irgend einer Zeit von ihr weiss. An ihre letzte "Idee" und deren vollendete Erfüllung ist dabei allein zu denken. 2. Muss man sich auch hier, auf diesem höberen Niveau, wie immer und uaberall der (schon wiederholt betonten) durchgängigen Korrelation und Funktionalbeziehung zwischen transzendentalem "Gedanken" ${ }^{28}$ ) und Gegenstand bew̧usst bleiben. Infolge dieser restringiert sich für den Standpunkt der Einzelwissenschaft, also "Fon unten her" gesehen, meine obige Behauptung über das Verhältnis von Transzendentalphilosophie und Einzelwișsenschaft dahin, dass in jeder Ėinżelw̌rissenschaft nưr so viel echtē Wissenschaftlichkeit stecke, als darin Gegenständlichkeit $=$ Wahrheit enthalten sẹi. Und das ist doch nichts besonders Päradoxes.

Aber eben diesem Problem "Gegenständlichkeit = Wahrheit" ist nur beizukommen vom tránsżendentalen Standpunkt aus. Hier enthüllt sich die Tragweite des oben von mir Ausgeführten, dass all das, was die Denkkweise des gewöhnlichen Lebens wie auch die der Ëinzelwissenschaften "Gegenștäñde" nennt, iñ der trianszendentalen Pröblemstellung̀ den Generalnenner oder gemeinsamen Exponenten "Giltigkeit" bekommt.

Von đièsem dürchgëhendèn Gesichtspunkt aus ist die Tranșżendentalphilosophie die Wissenschaft, in der die Teilbewasstseine von der Welt, gènannt Einzelwissenschaften, zụm einheitlịchen Sëlbstbew̌usstsein, und damit eben doch erst „zu sich selbst kommen.“ Darin liegt also nichts Mystisches und auch niehts spezifisch Heggelšches, sondern' es ist nur eíne ganz nüchterne, rein logische Konsequenz, wenn man einmạl die transzendentale Problemstellung zũ Ende deñkt.

Aber eines liegt allerdings darin, dass nämlich die Philosophie (als wissênsçháftlíche Transžendentalphilosophie) auch heute noch, wie zu Zeiten Platos, „die Königin der Wissenschaften“ ist, zwar in etwas andèrëm Sinne als bei Plato, dafür aber mit tieferer Begrühndung ihres Rechts auf den Thron, - und dabei in demselben Umfange: denn siè ist natürlich dies Selbstbewusstsein nicht bloss fürr die Wissenschaft, sondiern aùch für die Kunst, die Moral, die Religion, für alle Gebiete eines überempirischen Lebens. ${ }^{29}$ )

28) Vergl. Rickert, „Zwei Wege derErk.-theorie"(Kantstud. XIV) S. 195 ff.

29) Philosophie ist das wissenschaftliche Selbstbewusstsein der Vernunft, und damit der Kultur als deren empirischer Konkretion in der historischen Entwickelung. 
Was folgt aus dem allem für unser Problem? Eine selbstverständliche, aber wie das meiste logisch Selbstrerständliche psychologisch lange nicht gesehene Aufgabe, nämlich: dass die transzendentale Frage nach den logischen Möglichkeitsbedingungen wie für die Naturwissenschaft, so auch für die Geschichtswissenschaft prinzipiell und systematisch gestellt werden muss, die Frage nach ihren Voraussetzungen und ihrer Methode, durch die sie erst den Charakter der Wissenschaft erhält. Die gesamten hier liegenden Probleme sind bekanntlich erst in Fluss gekommen durch Windelbands Strassburger Rektoratsrede "Geschichte und Naturwissenschaft" (1894), und das die darin aufgeworfenen Probleme weiter führende Buch von Rickert „Die Grenzen der naturwissenschaftlichen Begriffsbildung", mit dem bezeichnenden Untertitel: „Eine logische Einleitung in die historischen Wissenschaften" (1896/1902).

Die geschichtsphilosophischen Probleme, um die es sich hier handelt, kann man bezeichnen als Geschichtșlogik. Die Zentral= frage ist die nach dem "Gegenstand der Geschichts wissenschaft "; das aber bedeutet für die transzendentale Problemstellung: die Frage nach den formalen Prinzipien, die die Geschichte als Gegenstand der Wissenschaft zu einer geltenden Einheit, zu einem einzigen in sich zusammenhängenden Ganzen machen, nach der Einheitsform des historischen Wissens. Vom Standpunkte des Subjekts aus gesehen lautet sie: Nach welchen Prinzipien verfährt das Erkennen, um aus dem scheinbar chaotischen Flusse der unmittelbar erlebten Wirklichkeit in all ihrer "extenșiv und intensiv unend-

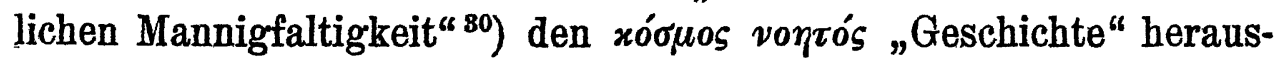
zustellen? Dabei wird zunächst, wie bei der transzendentalen Theorie der Naturwissenschaft, bloss die methodologische Struktur des fertig vorliegend gedachten historischen Wissens als eines vom Subjekt losgelösten, in sich selbst gründenden Zusammenhangs giltiger Sätze ins Auge gefasst, nịcht also die psychologische

30) Vergl. Rickert „Grenzen", S. 36. In diesem Ausgangspunkt liegt eine Berührung mit Bergson; nur dass, was bei R. bloss Ausgangspunkt ist, bei $B$. sich verabsolutiert und definitiv wird. B. gla ubt, diesen "Fluss der Erlebnisse" unmittelbar intuitiv erfassen zu können, wăhrend die Transzendentalphilosophie weiss, dass alles Erkennen (auch das intuitive, wenn es ein solches gibt), sofern es Geltung beansprucht, nach bestimmten begrifflichen Formen erfolgt, die und deren Geltungsgrund sich zum Bewusstsein zu bringen, eben das Geschäft der Transzendentalphilosophie ist. 
Genese des historischen Erkennens als eines Vorgangs in der Seele des Geschichtsforschers.

Hier könnte ein scheinbar ganz prinzipieller Einwurf gemacht werden: Ist denn die Geschichtswissenschaft überhaupt Wissenschaft? Es ist das im letztèn Grunde derselbe Knüppel, den man schon Kant und der ganzen Tranșzendentalphilosophie zwischen die Beine zu werfen versuchte, der Vorwurf: die Wissenschaft werde ja bei der ganzen Fragestellung schon vorausgesetzt, das ganze erkenntnistheoretische Unternehmen sei also zirkelhaft. ${ }^{81}$ )

Das klingt sehr einsichtig und ist sehr kurzsichtig; man glaubt da, vorurteilsfrei zu sein, und ist in Wirklichkeit nưr noch viel vorurteilsvoller und dogmatischer. Denn man misst dabei letzten Endes den Begriff der Wisssenschaft, der sich in der wissenschaftlichen Arbeit der Wissenschaft selbst herausbildet, an der aus den verschiedensten psychologischen Momenteń unterbewusst, vorwisssenschaftlich, unkritisch erwachsenen Vorstellung von Wissenschaft und Wahrheit, wie sie das gewöhnliche Leben und der sogenannte "gesunde Menschenverstand“" ${ }^{\text {82) }}$ haben: von dieser aus will man der Wišsenschaft sagen, was sie "eigentlich" zu wollen habe, während doch sonst in allen Fragen die Wissenschaft gérāde die Anfgabe hat, die unklaren und verworrenen Vorstellungsweisen des gewöhnlichen Lebens zu klären, zu korrigiereu, zu rekitifizieren. Die Wahrheit ist nirgends anders zu finden als in der Wissenschaft; also ist auch ihr Sinn und Begriff aus dieser herausžuarbeiten, an diesẹ zụm Bewusstsein zu bringen; nicht aber an einem mythologischen Phantasieprodukt und Wunschgebilde die wirklichen Wịssenschaften und ihre Leistungen zu messen.

Die Geschichtswissenschaft ist als Wissenschaft kulturwirklich; nicht mit einem von aussen, fremd an sie herangebrachten Wissenschaftsbegriff ist sie zu messen, sondern aus ihr selbst ist mittels dèr transzendentallogischen Sonde ihr begrifflicher Sinn als Wissenschaft heraúsz̃uhólen. Da aber zeigt sich: der platonisch-kantische Wissensbegriff, dạss es ein Wissen nur vom Generellen gebe, ist durch den Fortschritt des Kulturgeistes selbst überholt: es gibt

s1) woraus dann von selbst ${ }_{n}$ die Unmöglichkeit der Erkenntnistheorie" (Vortrag von Leonard Nelson auf dem Philosophen-Kongress in Bologna, gedrückt: Gö̈ttingen 1911) folge - natürlich nur des "sogenannten Erkenntnisproblems" (1908) wie es sich N. zurecht macht!

32) (F. Cassirer: „Der kritische Idealismus und die Philosophie des "gesunden Menschenverstandes", 1906.) 
auch eine Wissenschaft vom Individuell-Besonderen um seiner Besonderheit willen.'

Das nun ist das erste Problem der Geschichtslogik: Wie ist eine Wissenschaft vom Individuellen und Besonderen möglich, für welche die Individualität nicht bloss Exemplar und Experimentierobjekt des Allgemeinen ist? Wie kann es das geben? Welches ist der Sinn einer solchen Wissenschaft? Welches sind die $\mathrm{Zu}$ sammenhang konstituierenden Prinzipien der Auswahl und Neusynthese des "Wesentlichen"? Welehes die Kategorien der Geschichtswissenschaft zur Anordnung des Stoffes auf jene obersten Gesichtspunkte hin? Denn die konstitutiven Kategorien der Naturwissenschaft, Substanz und Kausalität, genügen in ihrem naturwissenschaftlichen Sinne nicht, um begreiflich zu machen, dass es eine einzige, einmalige Kette historischer Phänomene als einen Geltungszusammenhang sui generis gibt. Mittels ihrer kommt bloss eine "Natur" heraus, die ausnahmslos alles Geschehen umfasst; aber nicht alles Geschehen ist Geschichte: welches sind die Prinzipien, die aus der allgemeinen kausalgesetzlichen Verknüpfung aller Geschehnisse eine bestimmte Kette von Phänomenen als historische heraushebt, welches das "geist'ge Band", das diese Stücke zu einer sinnvollen, in sich geschlossenen Einheit macht?

Des Genaueren handelt es sich um zwei Grundprobleme: 1. das Problem des Wertes und der Wertbeziehung als den Konstituentien der Geschichtswissenschaft als Wissenschaft sui generis (im Unterschiede von der Naturwissenschaft), 2. das Problem der "historischen Kausalität" als des Konstituens derjenigen Wirklichkeitssynthese, die Funktion der Geschichtswissenschaft als Wissenschaft ist (gegenüber der Frage nach der Freiheit in der Geschichte selbst). Ich gehe auf diese Fragen selbst hier nicht weiter ein; nur als Probleme wollte ich sie anfzeigen Probleme, die in der Gegenwart lebhaft erörtert werden, nicht nur in den verschiedenen philosophischen Lagern, sondern auch von Männern der Spezialwissenschaft selbst ( Nationalökonomen, unter denen ich dort Eduard Meyer, hier Max Weber besonders hervorhebe - gegen Lamprecht). ${ }^{38}$ )

33) Als Literatur verweise ich noch anf: Rickert "Kulturwissenschaft und Naturwissenschaft", 2. Aufl., 1910; Baensch „Über historische Kausalität" (Kantstudien XVII, S. $18 \mathrm{ff}$ ); Sergius Hessen "Individuelle 
Die Geschichtslogik hat es mit dem Problem des "Gegenstandes der Erkentnis" (Rickert) der Geschichtswissenschaft zu tun; dieses Problem aber bedarf einer Ergänzung durch Untersuchung des Problems der "Erkenntnis des Gegenstandes" in der Geschichtswissenschaft. Hier handelt es sich nun um die oben zurückgeśtellte Frage nach den Vorgängen in der Seele des Geschichtsforschers bei seiner Arbeit, also um erkenntnispsychologische, bezw. erkenntnisphänomenologische Fragen. Hierzu sind besonders die Arbeiten Diltheys und seiner Schüler zu erwähnen, in denen sich über die Schwierigkeit des historischen "Verstehens" und die Mittel und Wege historischer Interpretation und Rekonstruktion feinsinnige Ausführungen finden. Auch Simmels hierhergehörigen Gedankengänge liegen mehr auf dem psychologischen als dem logischen Gebiete. Derartige geschichtspsychologische Untersuchungen können sich dann herab erstrecken bis in die tech. nischen Methoden und Hilfsmittel der Materialbehandlung durch den Historiker selbst, worüber ja die Geschichtswissenschaft in Bernheims "Lehrbuch der historischen Methode" eịn ausgezeichnetes Werk besitzt.

Ich wende miçh zur 3. Problemgruppe, den Problemen, an die man meistens zuerst denkt bei dēm Worte "Geschichtsphilo-, sophie". Ich will die hier auftauchenden Fragen - im Ünteir schiede zu der auf die Geschichtswissenschaft gerichtete Geschichtslogik - unter dem Namen "Geschichtsethik" zusammenfassen; die Bedeutung, den Sinn dieser Etikette werden die dazu gehörigen Ausführungen selbst klar machen.

Die. Geschichtswisseñschaft konstituiert sich zu in sich zusammenhängēnden Geltungseinheiten durch Beziehung der Phänomene auf als geltend hingenommene Werte. Diese Werte für den empirischen Histtoriker sind zunächst bloss als faktisch anerkannt vorausgesetzte Werte, wie etwa der Wert "Staat" oder "Kunst" oder "Wirtschaft". Diẹses Beziehẹn auf historische Werte gewährt aber "Objektivität" nuur, wenn es "gründet" in einer Bèzogenheit auf in sich selbst geltende Werte: die empirische Objektivität setzt voraus, dass Wert und Wertbeziehung im Gegenständlichen, d. h. der reinen Geltungssphäre verankert

Kausalität" (,Ërgänzungsheft der Kantstudien" No. 15); (von psychologistischer Seite): E. Spranger „Die Grundlagen der Gesehichtswissenschaft", Berilin 1905. 
sind. M. a. W.: Die empirisch-historische „Richtigkeit" setzt die überempirisch-überhistorische "Wahrheit" als den schlechthinigen Massstab voraus, nach dem sie „sich zu richten" hat. Die Geschichtswissenschaft ist logisch, wenn die Geschichte Logos hat. Erst dadurch wird aus dem Wert als psychischem und historischem Faktum ein Wert als Wert, ein Wert in sich, eine schlechthinigunbedingte absolute Giltigkeit. ${ }^{84}$ )

Das Gelten der Werte stammt also nicht aus der Geschichte, sondern verleiht vielmehr selbst erst dieser ihren Geltungscharakter. Aber das hindert nicht, dass wir uns diese Werte an der Geschichte

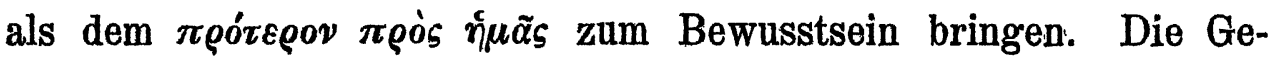
schichtswissenschaft selbst verfährt an Hand der empirischen Kausalität. Auch als "Universalgeschichte" ist sie ein kausales G efüge vom Standpunkte der positiven Wissenschaft. Aber über dem Gegenstand dieser Universalgeschichte erhebt sich die sachlichgeschichtsphilosophische Frage: welches ist in diesem empirischseienden Wertzusammenhang der schlechthin-geltende Sinnzusammenhang? Die Geschichtsphilosophie als wert-inhaltliche Disziplin hat den historischen Zeitzusammenhang der Inhalte in Stücke zu schlagen, um den überhistorischen Rangzusammenhang der Werte herausarbeiten zu können. $\mathrm{Zu}$ dem $\mathrm{Zwecke}$ hat sie eine Höhenwanderung vorzunehmen über die Gipfel der faktischen

34) Diese allein verdient den Namen "historischer Begriff" und steht als solcher auf logišsh gleicher Rangstufe wie die naturwissenschaftlichen Begriffe im strengen Sinne. [Vergl. über letztere: E. Cassirer "Substanzbegriff und Funktionsbegriff", (Berlin 1911).] Der historische Begriff aber unterscheidet sich vom naturwissenschaftlichen dadurch, dass die Selbstargumentierung der Geltungsfunktion hier nicht durch Selbstspezifizierung, d. h., von unten gesehen, durch "generalisierende", sondern durch „individualisierende Begriffsbildung“ erfolgt. [Vergl. die Problemstellung bei Friedr. Kuntze "Die kritische Lehre von der Objektivität", (Heidelberg, Winter, 1906), S. 303.] Um ein konkretes Beispiel zu geben:

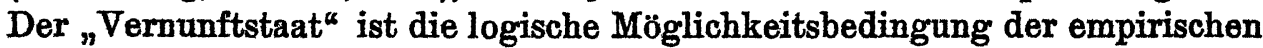
Staaten. In der Staatengeschichte kommt zu Bewusstsein und Realisation ein Stück Logos, nämlich der Staatsbegriff, wie er in der transzendentalen Sphäre des reinen Geltens konstituiert ist (seine Konstitution $=$ „Verfassung hat). Jener Vernunftbegriff ist aber für diese Entwicklung nicht bloss Ideal und Bewertungsnorm, sondern ihre Möglichkeitsbedingung, wodurch er erst zur giltigen Richtlinie wird. „Der Vernunftstaat ist selbst die vernünftige Wurzel der Existenz aller in historischer Erfahrung aufweisbaren Staaten (Medicus in der Einl. zu seiner Neuherausgabe von Fichte's "Geschlossenem Handelsstaat“, S. V.). 
historischen Entwickelung, um in einem Längsschnitt durch die Geschichte wertkritisch festzustellen, welche inhaltliche Erfüllung der vorausgesetzte ${ }^{85}$ ) Sinn der Menschheitsgeschichte überhaupt bisher gefunden hat. Während die empirische Geschichtswissenschaft restlos kausal orientiert ist, trägt diese philosophische Gèschichtstheorie teleologisches Gepräge.

Hier erhebt sich das schwere Problem der „historiokritizistischen" Methode - schwer zu bestimmen und noch schwerè zu handhaben - , wie aus dem Historischen das Überhistorische, aus dem zeitlichen Wëchs̀el das zeitlos B̉leibende heraũszupräparieren sei und zwar in wissenșchaftlicher, d. $h$. streng allgemeingiltiger Weise. Denn der Philosoph hat nicht die Werte zu "setzen": ${ }^{36}$ ) das gesehieht in den einzelnen Lebens-und Kulturgebieten durch deren Männer selbst, wohl aber sie in ihrer Geltung und ihrem Zusammenhang zu begründen. Soviel allerdings dürfte feststehen, dass "die Philosophie die Welt nicht synthetisch zu konstruieren, sondern nur analytisch zusammenżubuchstabierèn" hat. (Vergl. Windelbands Hegelrede, S. 14.) Aber um an den historisch vorliegènden Werten überhaupt nur eine Kritik üben zu können, muss sie sich leiten lassen von der - zwar an der Hand der Geschichte selbst, aber letžten Eindes doch nưr systematisch-konstruktiv zu gewinnenden - Idee eines letzten in sich abgeschlossenen Systems überhistorischer inhaltlicher Werte (als einem "Leitfaden a priori", wie Kant sagt), auf welches die gesamte historische Entwickelung in vielfach verschlungenen Wegen als sich hinbewegend anzunehmen ist, wobei der Gesichtspinkt der Annäherung an dies letzte System den Wertmassstab abgäbe, an dem die einzelnen historischen Werte zu messen sind. So entspringt hier aus der Geschichtsphilosophie selbst heraus das Problem der Transzendentalphilosophie als System überhaupt: Das „reine Gelten“

s5) Mit welchem Rechte ein solcher Sinn vorausgesetzt wird, ist in der 4. Problemgruppe abschliessend zu behandeln. Die sachliche Problemfolge innerhalb des Gesamtproblems "Geschichtsphilosophie“, der ich nachgehe, ist diese: 1. Wie ist Geschichtswissenschaft möglich? Antw.: Durch Werte als Konstituentien der historischen Wissenschaft. 2. Wie ist Gešchichte möglich? Antw.: Durch Werte als Konstituentien der historischen Wirklichkeit. 3. Wie sind absolute Werte und ihr Wirklichkeitszusammenhang möglich? Davon wird in dem letzten Abschnitt zu handeln sein.

se) Vergl. dagegen Riehl "Zur Einführung in die Phil. der.Gegenwart", 1. Vortrag, namentl. S, 22 ff. 
zerlegt sich für das in der historischen Entwickelung ${ }^{86 a}$ ) selbst darin stehende Subjekt in ein System absoluter Werte. "Die Totalität dieser Werte würde die abgeschlossene Wissenschaft von den Prinzipien des historisehen Universums sein und als eine ebenbürtige Schwester neben das System der Prinzipien des naturerklärenden Universums treten. Wie die Prinzipien des naturerklärenden Universums sich erfüllen in der „letzten Naturwissenschaft", so würden die Prinzipien des historischen Universums sich erfüllen in der Weltgeschichte". (Fr. Kuntze, loc. cit.).

Von hier begreift sich als notwendige Eigentümlichkeit der modernen Transzendentalphilosophie, woran ihre Gegner oft Anstoss genommen haben, nämlich 1. dass die Transzendentalphilosophie notwendig in eine sogenannte "historische Weltanschaunng" ausläuft, d.h. eine solche, die ihre Grundposition und ihr letztes Kreditiv nicht dem „Faktum der Vernunft“ ${ }^{37}$ ) "Natur", sondern dem „Faktum der Vernunft" „Geschichte" entnimmt, und 2. dass sie in all ihren Teilen "ethisches" Gepräge trägt - wenn man ethisch in dem ganz umfasșenden Sinne der Wertbezogenheit überhaupt nimmt. Der hierin sich begründende sogenannte „Primat der praktischen Vernunft" aber besagt nichts anderes als: a) Der Transzendentalphilosoph nimmt seine Grundstellung nicht im Seienden, sondern in der Geltungssphäre; b) diese aber wird für wertbewusste und wertwollende Subjekte zu einem "Reich absoluter Wertse", die in schöpferischer Tat der Synthesis zu Bewusstsein und Realisation zu bringen der letzte Sinn alles Menschenlebens ist.

Nun dürfte auch klar sein, warum ich die Gesamtheit der Probleme dieser 3. Gruppe als Geschichts ethik bezeichnet habe. Es rechtfertigt sich auch noch dadurch, dass diese Probleme schliesslich auch unmittelbar in den Dienst dessen treten, was man Ethik im engeren Sinne nennt. Jener Längsschnitt durch die Geschichte nämlich ergibt, wenn er gemäss der kritischen Wertung der einzelnen Etappen und deren Inhalte umrangiert wird, einen Querschnitt als normatives Wertesystem für die Gegen-

s6a) „Das historische Leben erhält sich nur dadurch abgesondert vom absoluten, dass es Kampf und Gegensatz zum Prinzip seiner Existenz hat" (Hans Ehrenberg "Die Geschichte des Menschen unserer Zeit", Heidelberg, 1911, im Nachwort, S. 44).

37) Vergl. Kant „Kr. d. p. V., S. 47. 
wart. Dieses aber erfïllt die Funktion, der rein formalen Ethik des reinen Pflichtbewusstseins einen Rahmen. inhaltlicher Wertbestimmungen $\mathrm{zu}$ geben, an denen sich das an sich autonome Gewissen in Konfliktsfällen orientieren kann. M. a. W.: Aus der Idee eines an Hand der Geschichte zu gewinnenden letzten Systems absoluter inhaltlicher Werte sind die materialen Prinzipien zu gewinnen, die das rein formale ethische Grundprinzip inhaltlich substruieren. Die reine Form des kategorischen Imperativs - der ja nichts anderes ist als der "Gedanke“ des Wërtes an sich in seiner Einbettung in das Bewusstsein eines endlichen und sinnlich-mitbedingten Subjekts - erfüllt sich inhaltlich dahín: Handle geschichtlich! D. h. handle so, dass Dein Handeln als positiver Wert in die Geschichtswissenschaft aufgenommen zu werden verdient, weil es einen Wert in der Geschichte selbst gesetzt hat; weil Du an Deiner Stelle und gemäss Deinem Sein die Ewigkeit in die Zeit verpflanzt hast.

Ich kenne bloss eînen modernen Versuch, den in Vorstehendem skizizierten Problemkomplex eines in sich geschlossenen Wertésystems ausführlich zû behandeln: Hugo Münsterbergs "Philosophie der Werte" (Leipzig 1908). Auch wenn man nicht mit allen Einzelausführungèi einverstanden ist und auch die Grundlegung wegen' ihres' Stecken-bleibens in der Wertung des Subjekts nicht füir ausreichend hält, muss man das Buch un der sy̆stematischen Kraft willen, die es bekundet, als bedeutende philosophische Leistũng anerkennen.. ${ }^{88}$ )

Wie bei der 2: Probleḿgruppe läuft auch hier neben der objektiv-transzendentalen eine subjektiv-psychologische Nebenstriömüng einher, die der Philossophie eine viel̆ bescheidenere Aufgabe stellt, als ich im Obigen gethan habe. Und wiederum ist es Dilthey und seine Schüler, die hier die Parole ausgeben. Nach ihnen erschöpft sich hier die Arbeit des Geschichtsphilosophen in einer Analyse der psychologischen Zusammeñhänge der historischen Ereignisse, in einer Eruierung des Verwobenseins der Persönlichkeiten uñ der Zeitströmungen in allen Feinheiten dieses Gewebes: das letzte Ziel ișt die Herausstellung der hier vorliegenden sozialund individualpsychischen "Strukturzusammenhänge" and Typen. Hier werden also die Werte nicht in ihrer Reinheit herauszustellen

38) Vergl. dazu auch desselben Verfassers „Grundzüge der Psychologie", Ba. I, 1900 . 
gesucht, sondern in ihrer Einbettung in die Gemüter der sie erlebenden und lebenden Subjekte und Zeiten. Es handelt sich also um Wertungen, nicht um Werte; es wird am Werte nur die dem Subjekt, nicht die dem reinen Gelten zugekehrte Seite bebehandelt und auch allein gesehen. Im Einzelnen bekundet sich darin ein eminentes „Verständnis" historischen „Nacherlebens", aber dafür eine um so geringere Kraft für die systematischen Aufgaben der Philosophie. Und dies Versagen in systematischer Hinsicht wird nưr notdûrftig dadurch maskiert, dass man diese Aufgaben überhaupt als nichtstellbare ablehnt, weil das Leben selbst absolut unsystematisch sei. Darin aber steckt ein falsches Ideal der Wissenschaft and der Philosophie: die - allerdings unerfüllbare - Idee einer Adaequation der Wissenșchaft an die Wírklichkeit in all der Mannigfaltigkeit ihres Flusses. Gerade diese aber ist durch die transzendentale Einsicht und Problemstellūng überholt: der philosophische Begriff hat nichts mit einer Wiedergabe oder gar Spiegelung des Erlebbaren zu thun; er gilt vom Erleben und Leben und für das Erleben und Leben. Derartige Geltungsvoraussetzungen macht aber auch alles Verstehen. Denn alles historische Verstehen setzt - logisch! mag es sich dessen bewusst sein oder nicht - das philosophische Begreifen, d. h. den Begriff voraus. Und darum ist die systematische Funktion all dieser historisch-psychologischen und phänomenologischen Untersuchungen doch letztlich nur die, in den Dienst der Transzendentalphilosophie zu treten.

Wir kommen zum 4. und letzten Problem: Welches ist das letzte Fundament der absoluten Werte? Welches ihr Verhältnis zum Wirklichen? Es ist die alte Plato-frage, wie das Gelten mit dem Seienden zusammenhängt, das Zeitlose mit dem Zeitlichen, ${ }^{39}$ ) die sich für die Geschichtsphilosophie des genaueren in die zwei Fragen zerlegt: nach dem letzten Wertgrund einerseits, nach den Trägern and Realisatoren der Werte in der Wirklichkeit andererseits. Wir wollen das erste Problem das meta-ethische, das letztere das meta-historische nennen; sachlich hängen beide anfs engste zusammen.

Kann man die absolute Geltung der Werte selbst wieder deduzieren? Antwort: Nein! Denn alle Deduktion setzt ein solch

39) Eine kurze Geschichte dieses Problems gibt N. v. Bubnoff: "Zeitlichkeit und Zeitlosigkeit", Heidelberg 1911. 
Absolutes schon voraus. Es zeigt sich also hier, auf dem Höhepunkt des transzendental-kritischen Regressus, eine eigentümliche Problemstruktur. Während bis zu diesem letzten Punkte ihrer Gedankenentwicklung die Transzendentalphilosophie immer bemüht sein muss, Geltungs - und Seinsfragen, quaestiones iuris und quaestiones facti, scharf auseinanderzuhalten, erhebt sich hier gerade die Frage nach dem Verhältnis beider, nach dem Band, das sie in der einen Welt zusammenbindet: die letzte quaestio iuris transzendentalis mündet aus in eine letzte quaestio facti transzendentalis. Und es kann nicht anders sein. Denn weiter hinauf, "nach oben" über die Geltungssphäre hinaus, um von da ein Deduktionsprinzip herabzuholen, geht es nicht mehr; es wäre auch nutzlos, da dann ja dieses Prinzip seinerseits wieder deduziert werden müsste und so in infinitum. So bleibt an diesem letzten Punkte nur eine Antwort vom Standpunkte des erlebenden Subjekts; aber nicht des individuellen, sondern des allgemeingültigen Erlebnisses. Denn das Grunderilebnis des Subjekts als Subjekt ist gar nicht, dass es diesen oder jenen Inhalt hat, sondern das Weltsinnerle b̆nis überhaupt. ${ }^{40}$ ) Weltsinnerlebnis und religiöses Erlebñis aber ist ein und dasselbe, und so vollendet sich das System der Transzendentalphilosophie in der kritischen Religionsphilosophie als dem Abschluss der Lebens- und Geschichtsphilosophie.

Hier schliesst sich der Riss zwischen Geltendem und Seiendem, indem das Funktionalverhältnis von Wert und Wirklichkeit, von Vernunft und Material, von Form und Inhalt ein unmittelbar erlebtes und gelebtes wird: auf das Ganze der Welt als Ganzes bezogen wird es zul dem Funktionalverhältnis zwischen „Subjekt ựberhaupt" und "Objekt überhaupt", wodurch beide ihren "Gegen"=sinn verlieren und in einheitlicher Durchdringung "konkrete Vernunft", "Wertwirklichkeit", Gott sind. Das einzelne religiöse Subjekt erlebt unmittelbar, d. h. ohne begriffliche Vermittelung, sein Eingereihtsein in den Weltsinnprozess überhaupt. Jede begriffliche Formulierung Gottes ist immer die eines endlichen Subjekts in dem Momente, wo es die letzte endliche Schranke abžulegen sucht: aber sie liegt dabei immer noch

40) Von hier aus gesehen ist die Aufgabe der Transzendentalphilosophie keine andere als die: das Weltsinnerlebnis zum Weltsinnbegriff zu klären. 
diesseits der Schranke der Endlichkeit. Der Gott, der „für uns“ gilt, ist nur, indem er Werte schaffend wird: der Gott "an sich" ist weder, noch gilt er, da beide Ausdrücke in ihrem Gegensinn sinnvoll sind nur durch die Subjektbezogenheit. ${ }^{41}$ ) Damit aber verliert all das Gerede vom "Gott draussen" und "Gott drinnen" ("Vernunft in der Welt" und „Vernunft im Einzelsubjekt") seinen Sinn, wenn damit ein Gegensatz statuiert sein soll.

Bei der Frage nach der Absolutheit der Werte tritt also an Stelle des logischen Denk-deductivis "von oben" das historische Lebenscreditiv „von unten“, aus -der Geschichte selbst heraus. Man kann Gott nicht beweisen wollen, da man inn ja dabei immer schon voraussetzt, sondern nur zum Bewusstsein bringen. Dass wir ihn aber als „Persönlichkeit " denken, besagt weiter gar nichts, als dass wir das Einheitsprinzip von Wert und Wirklichkeit, in dem beide ihre gemeinsame Wurzel und gegenseitige $A b$ stimmung auf einander haben, eben nicht als wertfremde, sondern als wertbezogene Weltordnung verehren : die universale Personalität als Einheitsprinzip des Universums als eines Kosmos von Werten. Die Naturwissenschaft aber und ihre Funktion, die "Natur", ist ein Wertkonstitut, wie alles Geltende, kann also so wenig die wertgetragene, d. h. religiöse Weltanschauung entthronen, dass sie vielmehr dieser zur Begründung ihres eigenen Geltens bedarf.

Das 2. Problem, das hier unmittelbar ansetzt und sich mit dem soeben behandelten verschlingt, ist, von dem soeben erreichten Standpunkt aus formuliert, die Frage nach der Entfaltung Gottes in der Geschichte, das Problem des "Reiches Gottes auf Erden", oder das Problem des Geistes. ${ }^{42}$ ) Hier handelt es sich um die Frage, ob den handelnden Subjekten der Geschichte nicht-bewusste Ideen, oder ob die wertbewussten und wertwollenden

41) Man kann das auch so ausdrücken: Gott selbst hat keine Religion. (Nebenbei bemerkt: In der Behandlung der von Troeltsch aufgeworfenen, neuerdings viel ventilierten Frage nach dem "religiösen Apriori" herrscht im Allgemeinen eine erhebliche Verschwommenheit bezüglich des Begriffs „Apriori“; ohne Klarheit hierüber kann es natïrlich nicht einmal zu einer richtigen Problemstellung kommen. Vergl. E. W. Ma y er in d. "Ztschr. f. Theol. u. Kirche", XXII, S. 59ff.)

42) Diesen Begriff sollte man begrifflich streng trennen von dem des Geltens, und nicht Probleme des Geisteslebens einerseits, Geltungsprobleme andererseits in einander fliessen lassen. Um Geistiges handelt es sich nur da, wo Wert und Wirklichkeit sich vereinen, wo es sich um werterfüllte Wirklichkeit handelt. . 
Subjekte die Träger und Realisatoren der Geschichte in ihrem letzten Wesensgehalte sind. In groben Umrissen gesehen ist es der Gegensatz: Hegel-Fichte. Aber wie doch eigentlich schon bei jedem dieser beiden (gleich grossen) grössten Kantianer in dem Ganzen ihrer Gesamtanschauung eine Synthese dieser beiden Momente vorliegt, so ist sie doch wohl auch das sachlich Begründete. Die freien Persönlichkeiten sind die Träger der Geschichte; die Freiheit zum Wert um des Wertes willen ist der Adel des Menschen und die Geschichte das Medium seiner Entfaltung. ${ }^{43}$ ) Aber die Freiheit zum Wert ist nicht möglich ohne den Wert selbst. Persönlichkeit ist das Subjekt nur als Realisator der absoluten

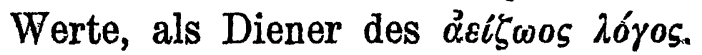

Zur erkenntnistheoretischen Erläuterung aber sei hierzu folgendes angemerkt: Das „Postulat der Begreiflichkeit der Natur" (Helṃholtz! Vergl. Kants „Prinzip der Zweckmässigkeị für unser Erkenñtnisvermögen" in Ansehung, des Problems „der Speżifikation der Natur". Kr. đ. U., Einl. S. XXXIV/XXXVII) erweitert sich konsequent zu dem ganz allgemeinen Postulat der Bègreifllichkeit des Welt- und Menschheitsgeschehens überhaupt, und von hier dann zu dem der Realisierbarkeit der Vernunft überhaupt, d. h. der absoluten Werte in der Wirklichlkeit überhaupt. Hegel streift nun, gerade auf Grund sèiner Geschichtsphiloșophie, diesem Prinzip den Postulatscharakter ab. Die Begreifliçhkeit der Welt begrẹift sich aus ihrer Begrifflichkeit, d. h. aus ihrem Angèlegtsein auf die absoluten Werte. Für die begrifflich erkennende Betrachtung ist dies ein "glücklicher Zufall" (Kant und Lotżè): der religiöse Mensch erlebt darin unmittelbar den Sinn der Welt und seiner selbst.

Damit hat sich uns der Kreis der Probleme geschlossen. Wènn ich zum Schluss nochmals die Fragge nach dem Verhältnis der Phillosophie zu den Einzelwissenschaften (urd Einzelkulturgebieten überhaupt) aufwerfe, so scheint es ein Verhältnis sowohl

43) Niicht in der rückwärtsschauenden Geschichtswissenschaftstheorie, sondern in der vor wäartsschauenden Geschichtstheorie hat diè "Freiheit des Menschen" ihre Stelle. Sie betrifft nicht den Unterschied von Geschichtswissenschaft und Naturwisssenschaft - wie Mïnsterberg und Medicus wollen - wohl aber den von Natur und Geschichte. [Zwischen beiden liegt als Bindeglied das "Problem des Lebens"; daraus begreift sich mein "rielleicht" auf S. 351]. 
der Abhängigkeit als der Unabhängigkeit za sein. Der Weg zum System geht zweifelsohne - analytisch! - von der Peripherie zum Zentrum, von den Einzelwissenschaften zu ihren letzten Gründen und Voraussetzungen; der Weg des Systems aber geht doch wohl - synthetisch! - vom Zentrum zur Peripherie. Nachdem wir den analytischen Weg von Problem zu Problẹm zụückgelegt, käme nun die Aufgabe, von rückwärts das Ganze als in sich geschlossene Einheit, synthetisch von einer Erkenntnis zur andern schreitend, nochmals zu bauen. Für unsern Problemzusammenhang also: Die und die Gründe geben einen absoluten Sinn der Welt und Menschheitsgeschichte, die und die Faktoren sind die Träger der historischen Entwickelung, auf das und das Wertesystem geht alles aus, in den und den Werten hàt es sich bis jetzt erfüllt; sie also sind die Richtlinien der Zukunftsarbeit und die synthetischen Prinzipien der Geschichtswissenschaft, und um dieser Richtlinien willen hat die Geschichtswissenschaft den und den Wert.

Aber trotzdem die Analysis die Synthesis logisch voraussetzt, ist uns in der Geschichte darin stehenden Wesen, ohne vorangegangene Analyse und Bindung an deren Resultate, dieser synthetische Weg (die via regia des "spekulativen Genies") nicht gangbar. Uns soll ebensowenig wie Zarathustra die eisige Ätherluft der Abstraktion den Atem beklemmen, uns soll vielmehr ebenso wie ihm ihre Reinheit und Schärfe, ja ihre Kälțe ureigenstes Lebenselenent sein. Aber nur wenn wir in mühsamem Aufstieg die Wege kennen gelernt und auf ihre Gangbarkeit geprüft haben, sind wir unseres Ganges sicher. Sonst passiert es leicht, dass die Erdennebel dem Gipfelwanderer den Blick trüben, die bösen Nebel, die uns die letzte Wabrheit intuitiv zu schauen verwehren: er stolpert, er stürzt und versinkt im Nebelmeer.

Vor die Philosophie als Wissenschaft haben die Götter den Schweiss gesetzt. 